\title{
Microseismic Event Estimation Based on an Efficient Wavefield Inversion
}

\author{
Chao Song (i) and Tariq Alkhalifah
}

\begin{abstract}
Full-waveform inversion (FWI) has shown its potentials in active seismic scenario in extracting a high-resolution velocity model of the subsurface. In the microseismic scenario, the source function is unknown, and FWI can be applied to optimize the source image and the velocity model, simultaneously. However, FWI is a highly nonlinear optimization problem, and it causes a bigger challenge when the source location and origin time are unknown. To mitigate these issues, we propose a two-stage scheme to invert for the source function and the velocity using an efficient wavefield inversion (EWI). We use outer-loop iterations to repeat the process until we achieve convergence. We specifically formulate an optimization problem to linearly reconstruct the wavefield that tries to fit both the data, as well as the wave equation corresponding to the background model. In the first stage, the reconstructed wavefield is used to calculate a source function using the background wave equation modeling operator without any inversion or update process. In the second stage, we use the computed source function to represent the true source to update the velocity model in the same way we use EWI in the active seismic case. Applications on data generated from a modified Marmousi model with a single microseismic event and multiple simultaneous events demonstrate the ability of the proposed method. An application to field dataset also demonstrates that the proposed method is effective in locating the microseismic event and inverting for a reasonably good velocity model, sequentially.
\end{abstract}

Index Terms-Cycle-skipping, efficient waveform inversion (EWI), frequency-domain, microseismic event estimation, signal reconstruction.

\section{INTRODUCTION}

$\mathbf{S}$ MALL earthquake events caused by human activities, such as mining, hydrocarbon extraction, and hydraulic fracturing are referred to as microseismic or induced seismicity. Microseismic event estimation helps engineers monitor the subsurface processes and map the anisotropy [1], [2]. Many methods have been developed to estimate microseismic events. Traveltimebased methods depend highly on the first arrival picking of $\mathrm{P}$ - and S-waves [3], [4]. Ray-based methods are also used to jointly locate the microseismic events and invert for the velocity for anisotropic medium [5], [6]. However, if the signal-to-noise ratio is low, the picking process becomes erroneous. As a result, the microseismic event detection and location are seriously

Manuscript received April 28, 2019; revised August 8, 2019 and September 29, 2019; accepted October 9, 2019. (Corresponding author: Chao Song.)

The authors are with the Physical Sciences and Engineering Division, King Abdullah University of Science and Technology, Thuwal 23955, Saudi Arabia (e-mail: chao.song@kaust.edu.sa; tariq.alkhalifah@kaust.edu.sa).

Color versions of one or more of the figures in this article are available online at http://ieeexplore.ieee.org.

Digital Object Identifier 10.1109/JSTARS.2019.2946903 degraded. Time-reversal-based methods [7]-[10] propagate the recoded data backward (reverse time) to reconstruct the wavefield using the wave equation. With an appropriate imaging condition, a source image can be obtained [11], [12]. This category of methods highly depends on the accuracy of the velocity model. The uncertainties of the source wavelet and the source-origin time also make the wavefield reconstruction more difficult [13]. Full-waveform inversion (FWI) has been adopted to invert for the source parts and the velocity model. FWI was applied to invert for the source components by separating the wave equation source term into spatial and temporal components under the condition we knew the true velocity [14]. To further improve FWI-based methods in microseismic, a source-independent FWI method was introduced to invert for the velocity model and the source components [15]. In the frequency domain, a source-extension approach was proposed to determine the source location while inverting for the velocity and source image, simultaneously [16]. To further improve this method, using the multiscattered energy in the data is able to provide better illumination to the velocity model when low frequencies in the data are missing [17]. They use a penalty term in the misfit function to enforce a focused source image. This penalty function, used to measure the source image focusing property, is applied as an objective function to optimize the source image and the velocity model [18]. Besides the source location and velocity inversion, FWI is also used to invert for the moment tensor [19], [20]. Most of the work mentioned above is based on the conventional FWI [21]. Conventional FWI is based on minimizing the misfit between the observed and predicted data in a least-squares sense subject to predicted data satisfying the wave equation. It is easy to fall into a local minimum using gradient-based optimization methods, and it will be a more severe problem when the source function is unknown in a microseismic scenario. Wavefield reconstruction inversion (WRI) was proposed to mitigate the cycle-skipping issue by using the wave equation as an additional regularization term, instead of a constraint [22]. This allows for a larger search-space over both the wavefield and the model, and it has been proved to be effective and efficient in the active seismic case.

To further improve WRI by including multiscattering components in the reconstructed wavefields efficiently, an efficient wavefield inversion (EWI) was proposed [23], [24]. As we calculate the wavefield with a least-squares approach, this method is capable of reconstructing the wavefield partially even if the source function is unknown as in the microseismic case. The reconstructed wavefield can be used to calculate the source 
function efficiently without any matrix inverse calculation. Thus, EWI potentiality can be useful for microseismic event estimation.

In this article, we use EWI to invert for the source function and velocity model in a two-stage scheme. To reduce the tradeoff between the source function and velocity model, we update these two parameters sequentially. We use an outer loop to repeat these two steps until the convergence is achieved. The outer loop is necessary to optimize the source function and velocity model, iteratively. As the wavefield in the model space can be reconstructed using an augmented wave equation, the unknown microseismic source function can be easily calculated without any inversion process. In this case, the velocity perturbation can be calculated via direct division. Application on a modified Marmousi model shows that the proposed approach can yield a reasonably good inverted velocity model, which can help locate microseismic events accurately. We also demonstrate the effectiveness of the proposed method with a field dataset.

\section{THEORY}

\section{A. Source Function Inversion}

In the conventional FWI implementation, we measure the misfit between the observed data and the predicted data from a wavefield that satisfies the wave equation, which can be expressed in short form as

$$
J(V)=\min \frac{1}{2}\|d-C u\|_{2}^{2} \quad \text { s.t. } \quad L(V, \omega) u=f(\mathbf{x}, \omega)
$$

where $d$ is the observed data, and $C$ is a mapping operator, which projects the wavefield to the receiver positions. We use $u$ to represent the wavefield, and $L(V)=\nabla^{2}+\left(V_{0}+V\right) \omega^{2}=$ $L_{0}+\omega^{2} V$ is the modeling operator, with the operator $L_{0}\left(V_{0}\right)=$ $\nabla^{2}+V_{0} \omega^{2}$ corresponding to the background squared slowness, $V_{0}$. We use $V$ as the squared slowness perturbation, and set it to zero at the beginning of the inversion process. As we implement this method in the frequency domain, the source origin time uncertainty is mitigated. The source function $f(\mathbf{x}, \omega)$, made up of complex values, includes both the wavelet and spatial information. The amplitude of $f(x, \omega)$ represents the spatial feature of the source function, which is referenced to as the source image $|f(\mathbf{x}, \omega)|$. Based on the adjoint-state method [25], the velocity and source function gradients are given, respectively, by

$$
\begin{aligned}
& \nabla_{V} J=\Re\left\{\omega^{2} u L^{-T}\left(C^{T} \Delta d\right)\right\} \\
& \nabla_{f} J=\sum_{\omega} \omega^{2} L^{-T}\left(C^{T} \Delta d\right)
\end{aligned}
$$

where $\Delta d=d-C u$ is the data residual, and $L^{-T}\left(C^{T} \Delta d\right)$ is the back-propagated wavefield. $\nabla_{V} J$ is given by elements that span the model space. The challenge in the conventional FWI is the large computational cost and the memory requirement in storing the background wavefields. The wavefield $u$ is calculated using the wave equation, which depends on the velocity. This dependency causes the nonlinearity of the objective function. That is because the wavefield is nonlinearly dependent on perturbations in the velocity. In order to solve these problems, WRI was proposed [22]. The optimization problem for WRI is given by

$$
E(V, u, f)=\min \frac{1}{2}\|d-C u\|_{2}^{2}+\frac{\epsilon}{2}\|L(V) u-f\|_{2}^{2} .
$$

In this new formulation, besides the data fitting term, the wave equation forms an additional regularization term. This approach requires the estimation of the wavefield in the whole model space first, then the model parameters can be updated. By inverting the synthetic wavefields and parameters, the search space is enlarged. In order to calculate the wavefield, which simultaneously satisfies the data fitting and the wave equation, we minimize $E(V, u, f)$ with respect to $u$ by solving $\nabla_{u} E(V, u, f)=0$. As a result, we calculate the wavefield by solving the augmented wave equation using the initial operator $L_{0}$

$$
\left(\begin{array}{c}
\epsilon L_{0} \\
C
\end{array}\right) u=\left(\begin{array}{c}
\epsilon f \\
d
\end{array}\right) \text {. }
$$

In the microseismic case, the source function is unknown. So we initialize the inversion with $f=0$. After obtaining the wavefield $u$, the source function is calculated by

$$
f_{0}(\mathbf{x})=\sum_{\omega} L_{0}(\omega) u(\omega)
$$

In (6), the final inverted source function $f_{0}(\mathbf{x})$ is obtained by stacking the inverted source functions for all frequencies. This will provide a source image focused mainly on the original source location, especially since the direct arrivals are often the strongest, and energy for the secondary sources pale in comparison. We use a source optimization function to force the source energy to focus in the source image. The source optimization function is given by [26]

$$
\begin{aligned}
J\left(f_{o p}, \mathbf{x}_{\mathbf{s}}\right)= & \frac{1}{2} \sum_{\mathbf{x}}\left\|f_{o p}(\mathbf{x})-f_{0}(\mathbf{x})\right\|_{2}^{2} \\
& +\frac{\alpha}{2} \sum_{\mathbf{x}}\left\|\left(\mathbf{x}-\mathbf{x}_{\mathbf{s}}\right) f_{o p}(\mathbf{x})\right\|_{2}^{2}
\end{aligned}
$$

where $f_{0}(\mathbf{x})$ is the input source function, and $f_{o p}(\mathbf{x})$ is the output optimized source function. $\mathbf{x}_{\mathbf{s}}$ denotes the estimated source location coordinate, and $\alpha$ is a weighting parameter controlling the focusing amount of the source function. The basic idea of (7) is to penalize nonphysical source energy not located at $\mathbf{x}=\mathbf{x}_{\mathbf{s}}$. This source penalty was originally proposed in an extended source function to replace the point source, with an aim to reduce cycle-skipping in FWI [27]. To calculate the estimated source location coordinate $\mathbf{x}_{\mathbf{s}}$, we set $\frac{\partial J}{\partial \mathbf{x}_{\mathbf{s}}}=0$ and get

$$
\frac{\partial J}{\partial \mathbf{x}_{\mathbf{s}}}=\sum_{\mathbf{x}}\left(\mathbf{x}-\mathbf{x}_{\mathbf{s}}\right) f_{0}(\mathbf{x})^{2}=0 .
$$

As a result

$$
\mathbf{x}_{\mathbf{s}}=\frac{\sum_{\mathbf{x}} f_{0}(\mathbf{x})^{2} \mathbf{x}}{\sum_{\mathbf{x}} f_{0}(\mathbf{x})^{2}}
$$

After obtaining $\mathbf{x}_{\mathbf{s}}$, we can optimize (7) by setting $\frac{\partial J}{\partial f_{o p}}=0$

$$
\frac{\partial J}{\partial f_{o p}}=f_{o p}-f_{0}+\alpha\left(\mathbf{x}-\mathbf{x}_{\mathbf{s}}\right)^{2} f_{o p}=0 .
$$


The optimized source function is given by

$$
f_{o p}(\mathbf{x})=\frac{f_{0}(\mathbf{x})}{1+\alpha\left(\mathbf{x}-\mathbf{x}_{\mathbf{s}}\right)^{2}} .
$$

\section{B. Velocity Inversion}

After inverting for the source image, we use it, given by $f_{0}(\mathbf{x})$ or $f_{o p}(\mathbf{x})$, to improve the velocity model as a second stage of the inversion. If we split the velocity model into background velocity and perturbation, the wave equation can be formulated as

$$
L u=f \rightarrow\left(L_{0}+\omega^{2} V\right) u=f \rightarrow L_{0} u=f_{e}
$$

where $f_{e}=f-\omega^{2} V u$ is a modified source function. Now we formulate an objective function independent of the velocity perturbation, given by

$$
E\left(u, f_{e}\right)=\min \frac{1}{2}\|d-C u\|_{2}^{2}+\frac{\epsilon}{2}\left\|L_{0} u-f_{e}\right\|_{2}^{2} .
$$

In (13), there are two independent variables: the wavefield $u$ and the modified source function $f_{e}$. The wavefield satisfies the following equation:

$$
\left(\begin{array}{c}
\epsilon L_{0} \\
C
\end{array}\right) \widehat{u}=\left(\begin{array}{c}
\epsilon f_{e} \\
d
\end{array}\right) .
$$

In solving for the wavefield, the operator $L_{0}$ remains stationary during the update process within each frequency. We use $\widehat{u}$ to represent the wavefield calculated from the inverted source function, which differs from $u$ (wavefield calculated for the source image inversion). In the beginning of velocity inversion, the velocity perturbation $V$ is set to zero, and we begin the inversion process with $f_{e}=f_{0}$. The modified source function is updated by

$$
f_{e}=L_{0} \hat{u} .
$$

Here, this $f_{e}$ will include the velocity perturbations, and we plug $f_{e}$ into (14) to perform inner iterations between (14) and (15) to add multiscattering components into $\widehat{u}$. Inner iterations is a key step in EWI, which is discussed in detail in [24]. After obtaining the reconstructed wavefield $\widehat{u}$ and the modified source function $f_{e}$, the velocity perturbation is calculated using direct division

$$
V=\frac{\left(f_{0}-f_{e}\right)}{\omega^{2} \widehat{u}} .
$$

To make the division operation more stable, we use a stabilizing term in the denominator

$$
V \approx \frac{\left(f_{0}-f_{e}\right) \widehat{u}^{*}}{\omega^{2} \widehat{u} \widehat{u}^{*}+\lambda}
$$

where $\lambda$ is a small real positive number to avoid dividing over zero and $*$ symbol denotes the complex conjugate. After obtaining the squared slowness perturbation $V$, we update the background model by

$$
V_{0}=V_{0}+V .
$$

In each selected frequency, we calculate the squared slowness perturbation and update the background operator $L_{0}$ and, then,

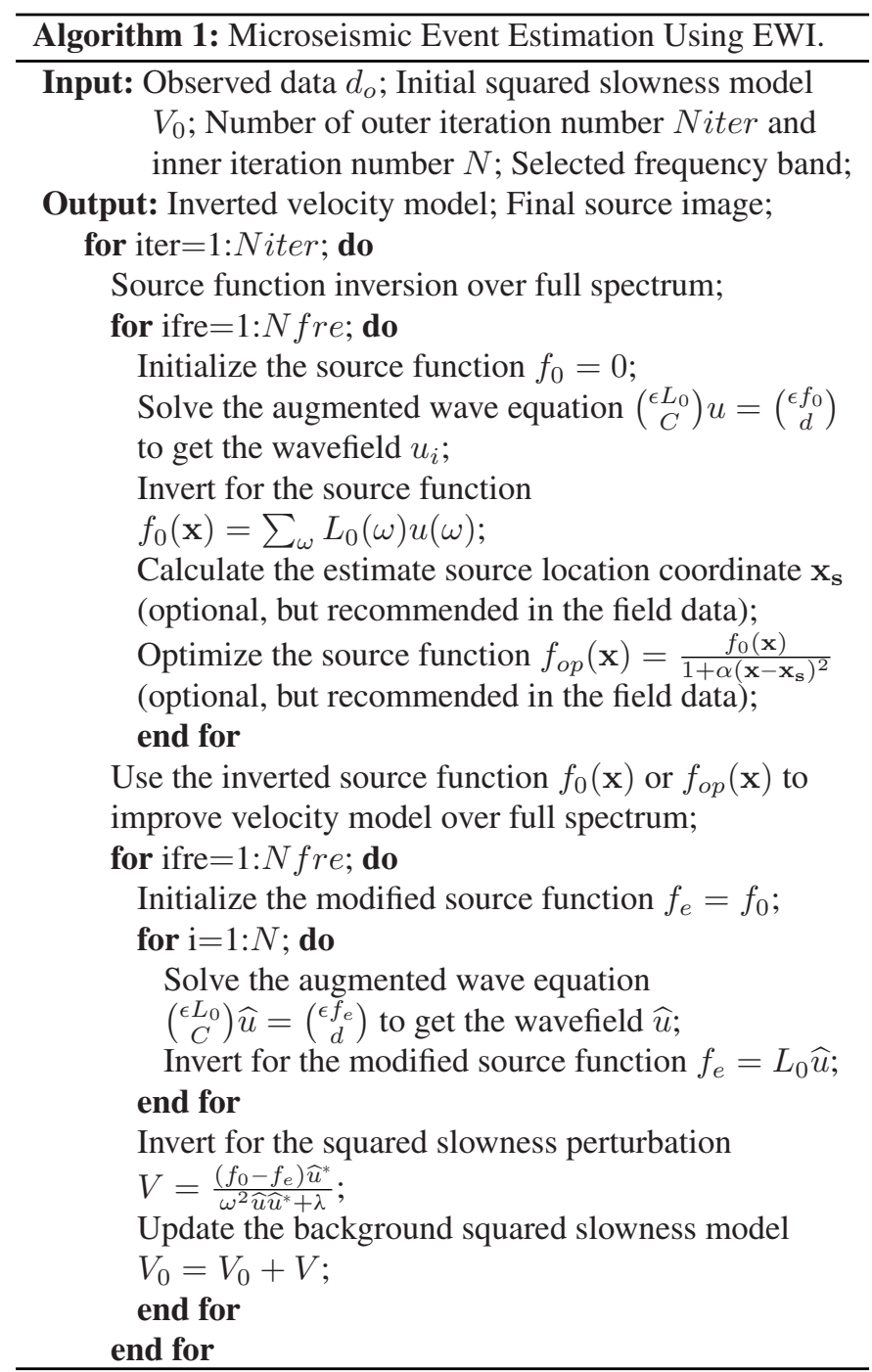

we move to the next frequency. The conventional FWI and the WRI implementations in the frequency domain perform many iterations in each frequency to update the model. EWI method is capable of calculating the velocity perturbation directly for each frequency with limited inner iterations (no matrix inversion) [24]. Thus, this method is simple and efficient. We need to use an outer loop to repeat the two-stage updating scheme over the full spectrum. The details of the algorithm are explained in Algorithm 1.

\section{NUMERICAL TEST}

\section{A. Homogeneous Model}

We first consider a homogeneous model of size $200 \times 200$ samples. We set the source location in the middle of the model, and 200 receivers on the surface given by the $\triangle$ symbol shown in Fig. 1. The $\epsilon$ we use in all examples in this article is $10 e^{7}$, and we choose this value based on trial and error tests. We perform the source function inversion starting from 3 to $8 \mathrm{~Hz}$ with a frequency interval of $0.5 \mathrm{~Hz}$. Following the source function inversion scheme introduced in the theory section, we set the 


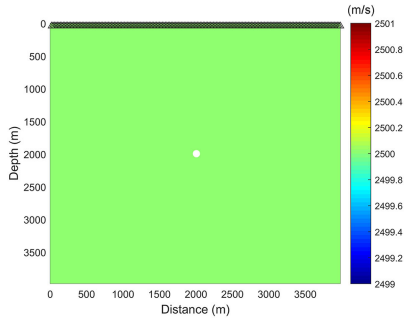

Fig. 1. Homogeneous velocity model with receivers on the surface (the white dot denotes the true source location).

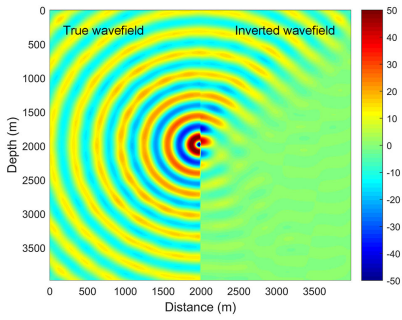

Fig. 2. True and inverted wavefields using the true velocity.

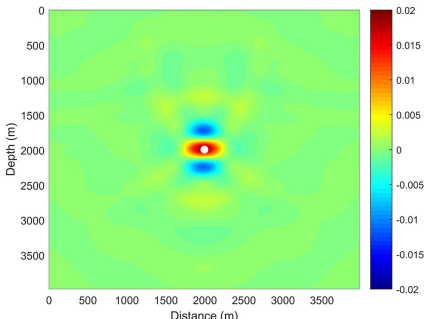

(a)

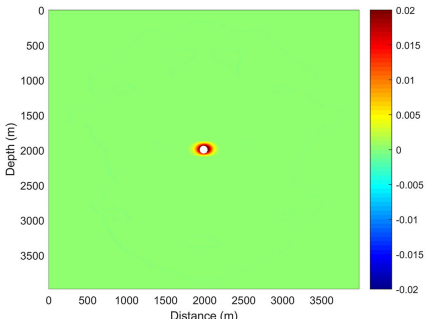

(b)
Fig. 3. Original inverted source image (a) and optimized source image (b) using the true velocity (the white dot denotes the true source location).

initial source function to zero. The true wavefield and inverted wavefields obtained from (4) at $8 \mathrm{~Hz}$ are shown in Fig. 2. As the data are only recorded on the surface, the reconstructed wavefield is distributed near the surface. The inverted source image is shown in Fig. 3(a), and the optimized source image is shown in Fig. 3(b). We see that the source energy in the original inverted source image is well focused on the true source location. However, there are some artifacts around the source location. After optimizing the original inverted source image using (9) with $\alpha=0.01$, the source energy is better focused and the artifacts are suppressed.

\section{B. 2-D Marmousi Model}

We further apply the proposed method on a modified Marmousi model of size $370 \times 80$ samples. The true velocity model is shown as in Fig. 4. The true source location coordinate is $(3700 \mathrm{~m}, 1000 \mathrm{~m})$ identified by the white dot. The horizontal and vertical grid interval is $20 \mathrm{~m}$. We start the inversion here from 3 up to $20 \mathrm{~Hz}$ with a frequency interval being $0.5 \mathrm{~Hz}$. We use a linearly increasing velocity with depth as the initial velocity model, shown in Fig. 5(a). Using the initial velocity

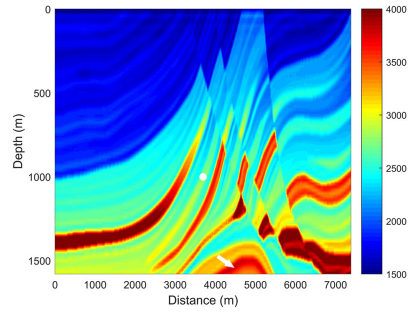

Fig. 4. True modified Marmousi model with a single source (the white dot denotes the true source location).

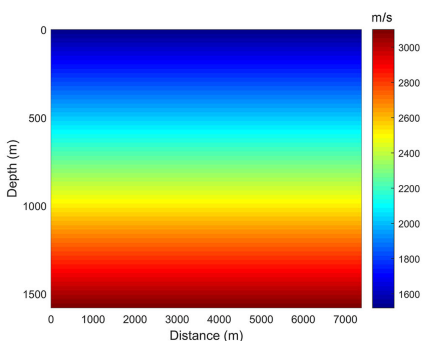

(a)

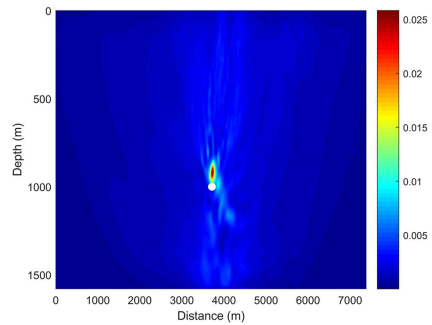

(b)
Fig. 5. Initial velocity model (a) and the EWI inverted source image using the initial velocity model (b) (the white dot denotes the true source location).

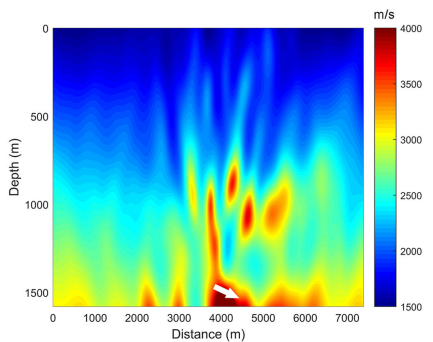

(a)

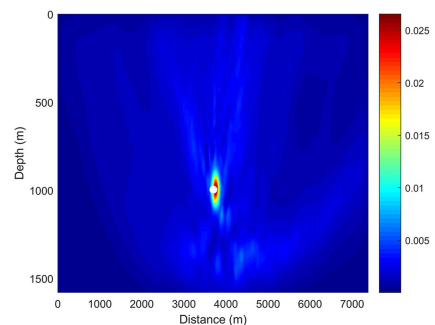

(b)
Fig. 6. EWI inverted velocity model (a) and final source image using inverted velocity model (b) with single source (the white dot denotes the true source location).

model, the inverted source image is shown in Fig. 5(b). As the initial velocity model is far from the true one, the source energy is not well focused at the true source location. In each selected frequency, we perform two inner iterations. After 30 outer iterations, the inverted velocity model is shown in Fig. 6(a). It is obvious that the inverted velocity model captures some of the kinematic features of the true Marmousi. The inverted source image is shown in Fig. 6(b). The source energy is generally focused to the true source location. To demonstrate the superiority of the proposed method, we implement FWI to invert for the source image and velocity with the same inversion setup. The inverted velocity and the source image using FWI are shown in Fig. 7(a) and (b), respectively. From Fig. 7(b), it is obvious that the source image using FWI has lower resolution than that from EWI. The source energy is not fully focused to the true source location. In the microseismic scenario, the sources are located in the subsurface, while the receivers are usually distributed on the surface or in a monitoring well. As a result, the velocity update using waveform inversion is mainly contributed by the transmission wave. Due to the limited number 


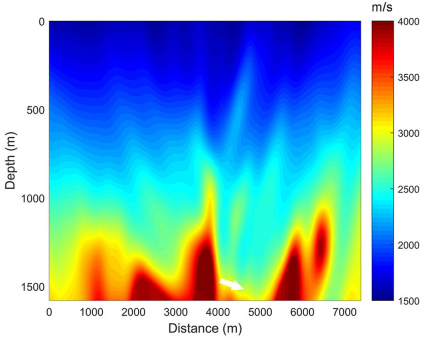

(a)

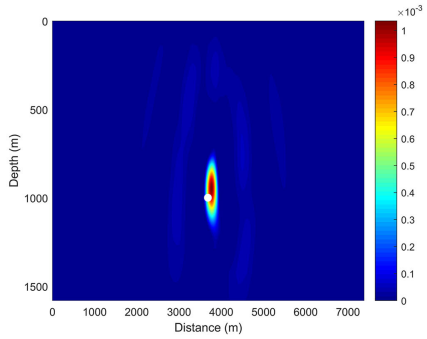

(b)
Fig. 7. FWI inverted velocity model (a) and final source image using inverted velocity model (b) with single source (the white dot denotes the true source location).

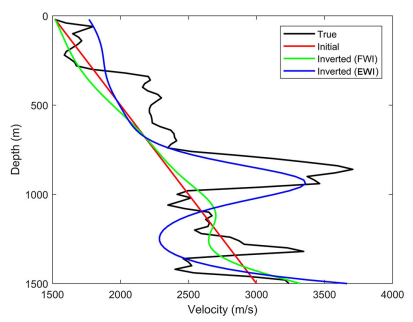

Fig. 8. Velocity profile at $4000 \mathrm{~m}$.

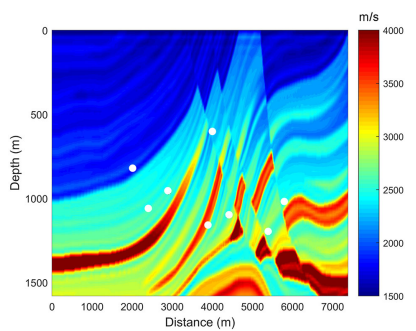

Fig. 9. True modified Marmousi model with eight sources (the white dots denote the true source locations).

of sources and the microseismic acquisition system, mainly low wave number components of the velocity model can be recovered, and this kinematically correct velocity model is good enough to accurately locate the microseismic events. From the velocity profile shown in Fig. 8, we see that the inverted velocity using EWI recovers the high-velocity area in the true model at a depth of $900 \mathrm{~m}$, and it fits the true one reasonably well. By comparison, FWI fails to capture the high-velocity area. There is a high-velocity dome in the deep part of the velocity model as the white arrow points out in Fig. 4. In the inverted velocity by FWI, Fig. 7(a) shows clear sign of cycle-skipping in the deep part. While EWI captures the high velocity in the deep part, as shown in Fig. 6(a).

If there are many microseismic events in the same survey area, and they overlap, we will need to deblend them. Specifically, we need to apply our method one event at a time. In this test, we set eight microseismic events randomly distributed in the survey area, as shown in Fig. 9. With the same inversion setup as the previous example, the EWI inverted source image for all the sources using the initial velocity is shown in Fig. 10. It is obvious that the source energy is poorly focused to the true source locations. Thus, we perform the EWI inversion for the

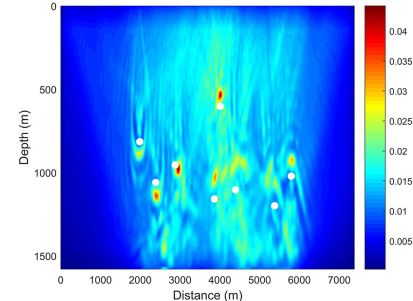

Fig. 10. EWI inverted source image using the initial velocity model (the white dots denote the true source locations)

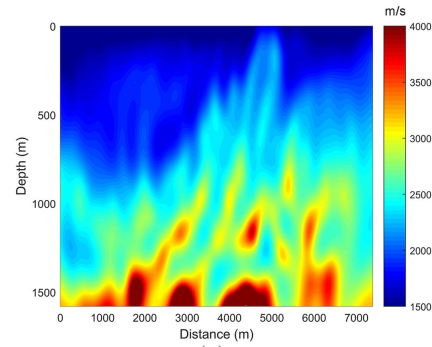

(a)

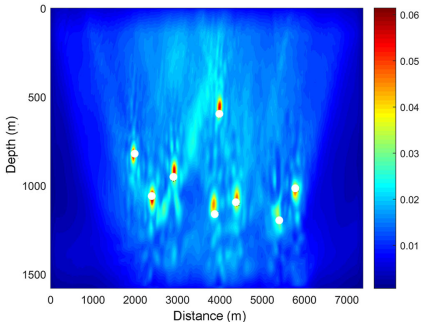

(b)
Fig. 11. EWI inverted velocity model (a) and final source image using inverted velocity model (b) with eight sources (the white dots denote the true source locations).

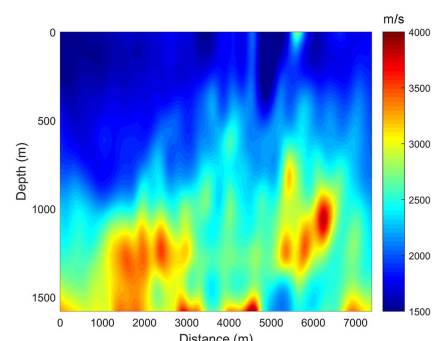

(a)

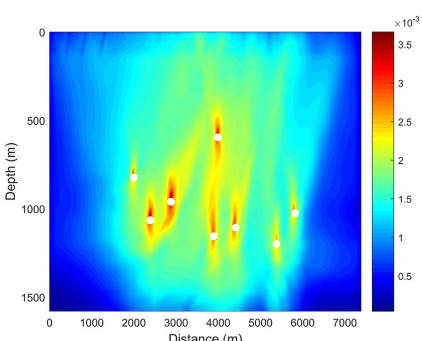

(b)
Fig. 12. FWI inverted velocity model (a) and final source image using inverted velocity model (b) with eight sources (the white dots denote the true source locations).

source function and velocity for all the sources, sequentially. The final inverted velocity and the source image are shown in Fig. 11(a) and (b), respectively. As we are using more sources to invert for the velocity, the illumination is better resulting in more coverage of the inverted velocity. As a result, we are able to obtain a reasonably good inverted velocity model, which resembles a smooth version of the true model. The source energy is accurately focused on the true source positions as the velocity improves. We also show the FWI final inverted velocity and source image using the same inversion setup in Fig. 12(a) and (b), respectively. We observe that EWI does better than FWI in inverting for the velocity and source image.

Next, we use two sources ignited simultaneously, which are located at $(3700 \mathrm{~m}, 1000 \mathrm{~m})$ and $(3700 \mathrm{~m}, 1400 \mathrm{~m})$ as white dots indicated in Fig. 13. In this example, we still use the linearly increasing with depth model, which is shown in Fig. 5(a). The inverted source image corresponding to the initial velocity model is shown in Fig. 14. We observe that the source energy is not focused at the true source locations. To improve the source 


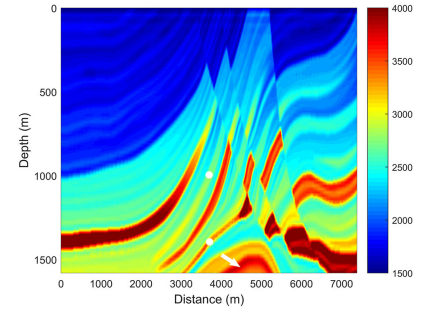

Fig. 13. True modified Marmousi model with two sources (the white dots denote the true source locations).

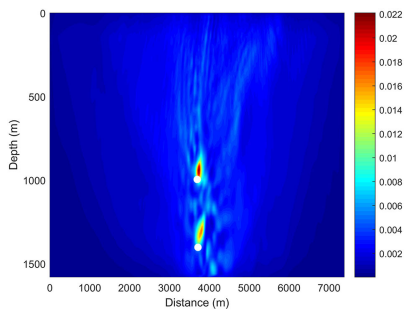

Fig. 14. EWI inverted source image using the initial velocity model with two simultaneous sources (the white dots denote the true source locations).

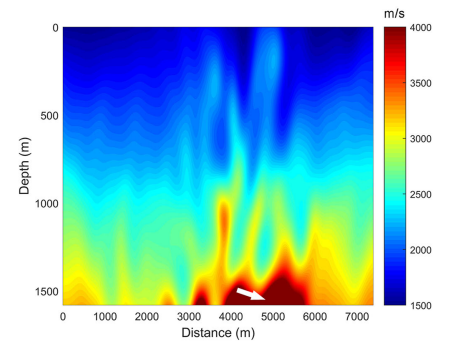

(a)

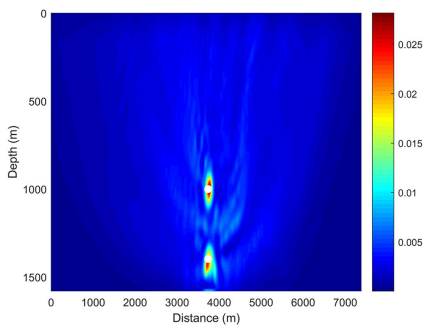

(b)
Fig. 15. EWI inverted velocity model (a) and final source image using inverted velocity model (b) with two simultaneous sources (the white dots denote the true source locations).

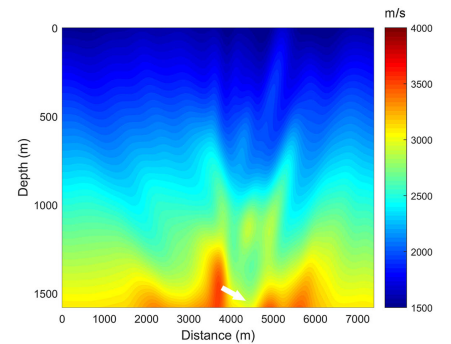

(a)

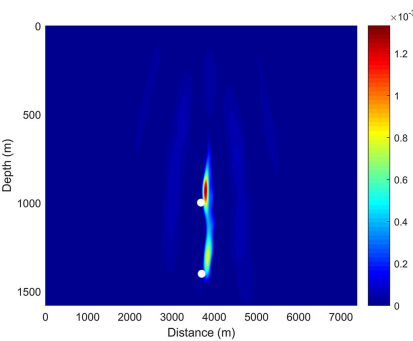

(b)
Fig. 16. FWI inverted velocity model (a) and final source image using inverted velocity model (b) with two simultaneous sources (the white dots denote the true source locations).

image quality, we perform 30 outer iterations of the simultaneous inversion for the source image and the velocity model. The inverted velocity model and source image are shown in Fig. 15(a) and (b), respectively. As the inversion is dominated by transmissions, the kinematic features of Marmousi model is reasonably recovered in the inverted model, and the source energy in the source image is focused very close to the true source locations. By comparison, the inverted velocity and the source image using FWI for multisource case are shown in Fig. 16(a) and (b), respectively. From Fig. 16(b), it shows that the source

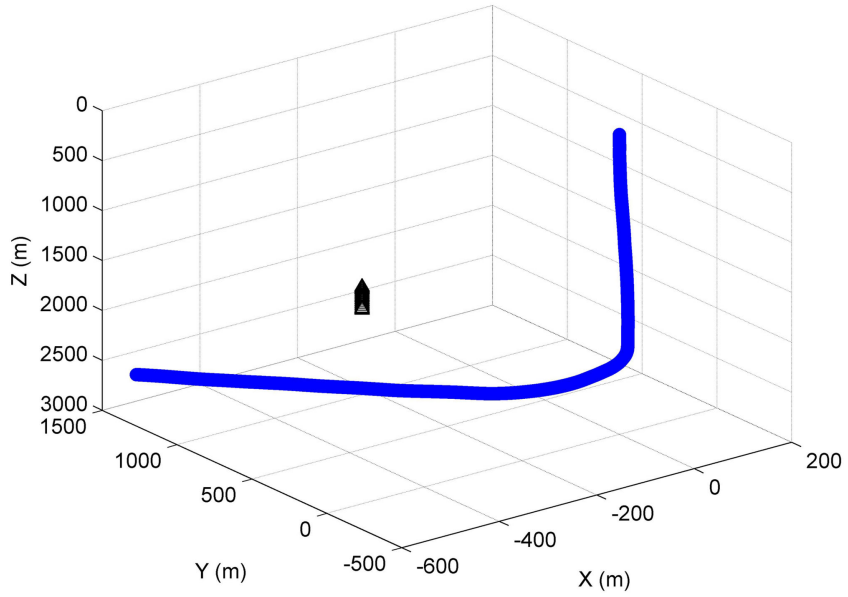

Fig. 17. Acquisition geometry with a treatment well (given by blue dots), and the receivers (given by black triangles).

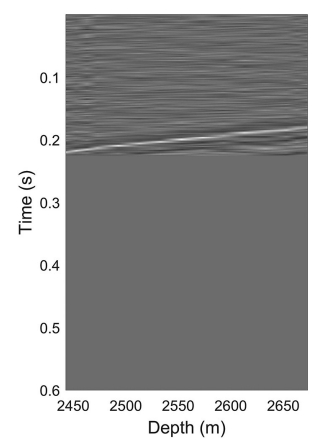

Fig. 18. Original data with the first arrivals afterwards muted.

energy of two microseismic events cannot be distinguished. It is difficult to locate these two microseismic events precisely using this low-resolution source image. In the simultaneous source test, FWI still fails to recover the high velocity in the deep part, as the white arrow in Fig. 16(a) shows. It is an indication that FWI falls into a local minimum. By comparison, the inverted velocity model using EWI is able to capture the high velocity in the deep part.

\section{2-D Real Data}

We finally test our proposed method on a single microseismic event captured in the field. This field dataset is collected from an oil field in China during an 11-stage hydraulic fracturing treatment. The recording system used in this survey consists of 15 levels of three-component geophones. The survey, which includes a treatment well (blue dots), a monitoring well (red dots), and receivers (black triangles) is shown in Fig. 17. We focus on the single event in Fig. 18, and muted all the arrivals afterwards. We choose one trace of the recorded data and analyze the frequency content. The trace and its frequency spectrum are shown in Fig. 19(a) and (b), respectively. We apply a bandpass filter between 10 and $80 \mathrm{~Hz}$. The filtered data spectrum and data are shown in Fig. 19(c) and (d), respectively. We use a homogeneous velocity model of $4000 \mathrm{~m} / \mathrm{s}$ as the initial velocity. The source image corresponding to the initial velocity model is shown in Fig. 20. As the initial velocity is very bad, the 


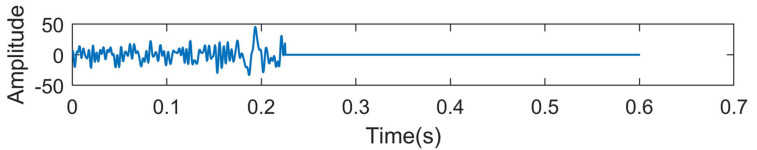

(a)

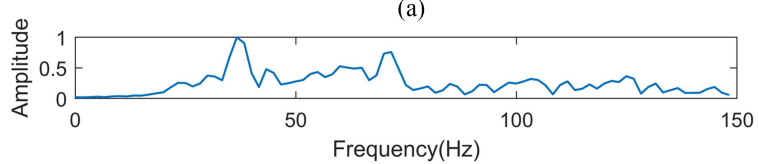

(b)

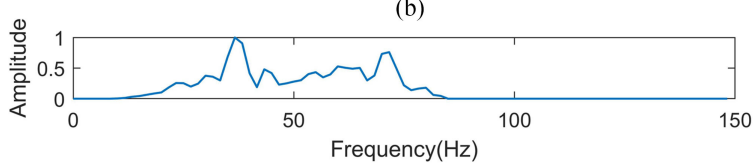

(c)

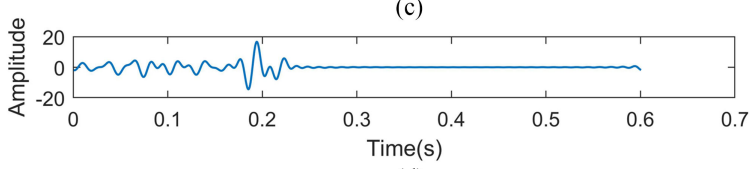

(d)

Fig. 19. One trace of the data at the eighth receiver (a), the frequency spectrum of this trace (b), the frequency spectrum after a bandpass filter (c), and the corresponding trace (d).

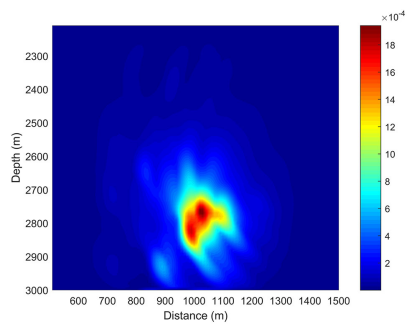

Fig. 20. Inverted source image using the initial velocity model.

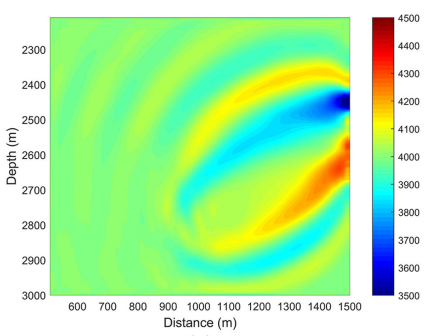

(a)

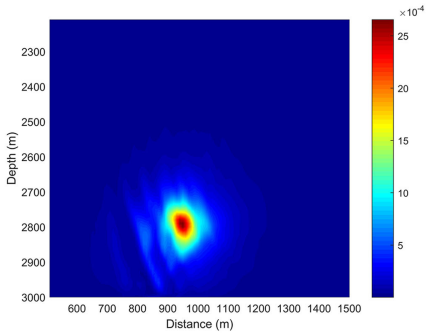

(b)
Fig. 21. Inverted velocity model (a), and the final inverted source image (b).

corresponding source image has a poor focusing feature, even though we use the optimized source function with $\alpha=0.001$. And it is difficult to determine the true source location.

As low-frequency components in the data are missing, we conduct the inversion process from 10 to $80 \mathrm{~Hz}$ with a sampling interval of $1.5 \mathrm{~Hz}$. In each selected frequency, we use two inner iterations. After 20 outer iterations of velocity update corresponding to the number of frequency bands, the inverted velocity is shown in Fig. 21(a). It is obvious that there is a low-velocity zone in the survey area. It is reasonable as the lowvelocity zone corresponds to the fluid injection well location. The inverted source image obtained from the inverted velocity model is shown in Fig. 21(b), and the source energy is focused a lot better compared with the source image we get from the initial

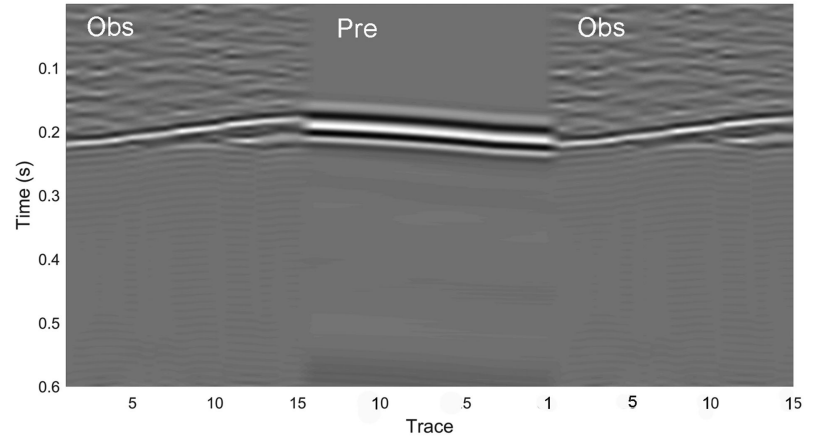

Fig. 22. Bandpass filtered data (left and right), and the predicted data (middle) after one outer iteration.

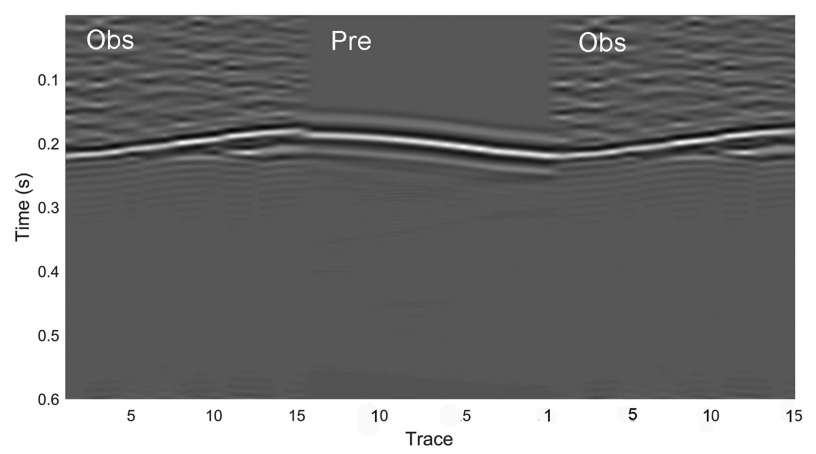

Fig. 23. Bandpass filtered data (left and right), and the predicted data (middle) after 20 outer iterations.

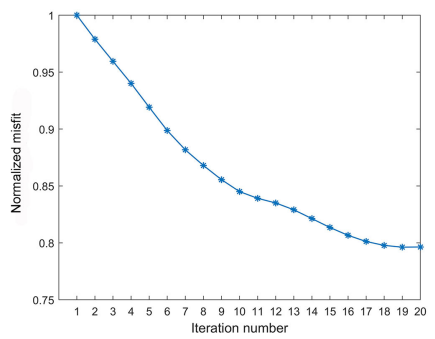

Fig. 24. Misfit as a function of 20 outer iterations.

velocity. We show the filtered observed and predicted data after one iteration in Fig. 22, and there are clear mismatches. After 20 outer iterations, as the velocity and source image improves, the predicted data fit the true filtered data reasonably well, which is shown in Fig. 23. The predicted data are obtained by time-domain modeling using the inverted velocity and source location. Considering that the normalized data misfit decreases reasonably well as shown in Fig. 24, the inversion process is converging.

\section{DISCUSSION}

We use an EWI to estimate the source location and improve the velocity model in a two-stage scheme. In the first stage, we set the initial source function to zero, and reconstruct the wavefield near the sensors' locations. With the reconstructed wavefield, the source function can be calculated efficiently. In the second stage, we use the inverted source function to perform the EWI velocity inversion like what is done in the active seismic case [28]. We 
use outer iterations to repeat this two-stage inversion, and the source function and the velocity are optimized iteratively until convergence is achieved.

In the velocity inversion, EWI uses a modified source $f_{e}$ to absorb the velocity perturbation. Inner iterations between $\hat{u}$ and $f_{e}$ inject multiscattering components to the reconstructed wavefield, $\hat{u}$, and multiscattering energy in the $\hat{u}$ will provide a better illumination of the velocity model [17]. Theoretically, the more inner iterations, the higher order scattering information is included in the wavefield. However, energy corresponding to multiscattering higher than the second order will barely contribute the velocity update [29], so two inner iterations are used in our implementations.

In the real data test, we recommend to use the source function optimization. Because it is able to provide a reasonably good source image. However, it may be dangerous to use a large $\alpha$, as it may enforce the source energy to the wrong source location. We only have one microseismic dataset to prove the validity of our proposed method, and we will appreciate anyone who wants to share data for research collaborations.

\section{CONCLUSION}

We introduced a new microseismic event estimation method based on an EWI. This new method is reasonably immune to the cycle-skipping problem, as well as a bad initial guess of source location often impeding FWI implementations. It also mitigates uncertainties of the source wavelet and the origin time in microseismic event estimation. We use the augmented wave equation to reconstruct the wavefield and calculate the source function. We apply a source optimization problem to get a focused source image. Then, we proposed to use the EWI method to optimize the velocity model in an efficient way. Applications to data generated from a modified Marmousi with a single source and multiple sources yield reasonably good results. The application on field data also shows that the proposed method can get reasonable velocity inversion results and improve the source image quality.

\section{ACKNOWLEDGMENT}

The authors would like to thank KAUST for its support and the SWAG group for the collaborative environment. They would also like to thank Yuyang Tan of USTC for sharing and processing the field data. This article utilized the resources of the Supercomputing Laboratory at King Abdullah University of Science and Technology (KAUST) in Thuwal, Saudi Arabia, and the authors are grateful for that.

\section{REFERENCES}

[1] N. Warpinski, "Microseismic monitoring: Inside and out," Soc. Petroleum Eng., vol. 61, pp. 80-85, 2009.

[2] P. Shi, S. Yuan, T. Wang, Y. Wang, and T. Liu, "Fracture identification in a tight sandstone reservoir: A seismic anisotropy and automatic multisensitive attribute fusion framework," IEEE Geosci. Remote Sens. Lett., vol. 15, no. 10 , pp. $1525-1529$, Oct. 2018.

[3] F. Waldhauser and W. Ellsworth, "A double-difference earthquake location algorithm: Method and application to the Northern Hayward Fault, California," Bull. Seismological Soc. Amer., vol. 90, no. 6, pp. 1353-1368, 2000.
[4] L. Eisner, P. M. Duncan, W. M. Heigl, and W. R. Keller, "Uncertainties in passive seismic monitoring," Leading Edge, vol. 28, pp. 648-655, 2009.

[5] J. Li, H.Zhang, W. L. Rodi, and M. N. Toksoz, "Joint microseismic location and anisotropic tomography using differential arrival times and differential backazimuths," Geophys. J. Int., vol. 195, no. 3, pp. 1917-1931, 2013.

[6] J. Li, C. Li, S. A. Morton, T. Dohmen, K. Katahara, and M. Nafi Toksöz, "Microseismic joint location and anisotropic velocity inversion for hydraulic fracturing in a tight bakken reservoir," Geophysics, vol. 79, no. 5, pp. C111-C122, 2014.

[7] D. Gajewski and E. Tessmer, "Reverse modelling for seismic event characterization," Geophys. J. Int., vol. 163, pp. 276-284, 2005.

[8] B. Artman, I. Podladtchikov, and B. Witten, "Source location using time-reverse imaging," Geophys. Prospecting, vol. 58, pp. 861-873, 2010.

[9] P. Sava, "Micro-earthquake monitoring with sparsely sampled data," $J$. Petroleum Exploration Prod. Technol., vol. 1, pp. 43-49, 2011.

[10] F. Li, Y. Qin, and W. Song, "Waveform inversion-assisted distributed reverse time migration for microseismic location," IEEE J. Sel. Topics Appl. Earth Observ. Remote Sens., vol. 12, no. 4, pp. 1327-1332, Apr. 2019.

[11] N. Nakata and G. C. Beroza, "Reverse time migration for microseismic sources using the geometric mean as an imaging condition," Geophysics, vol. 81, pp. 51-60, 2016.

[12] D. Rocha, P. Sava, J. Shragge, and B. Witten, "3d passive wavefield imaging using the energy norm," Geophysics, vol. 84, no. 2, pp. KS13-KS27, 2019.

[13] C. Song, T. Alkhalifah, Z. Wu, and B. Sun, "Non-stationary filter used in micro-seismic source imaging," in Proc. 87th Annu. Int. Meet., SEG, Expanded Abstr., 2017, pp. 2898-2902.

[14] J. Kaderli, W. D. McChesney, and S. E. Minkoff, "Microseismic event estimation in noisy data via full waveform inversion," in Proc. 85th Annu. Int. Meet., SEG, Expanded Abstr., 2015, pp. 1159-1164.

[15] H. Wang and T. Alkhalifah, "Microseismic imaging using a source function independent full waveform inversion method," Geophys. J. Int., vol. 214, no. 1, pp. 46-57, 2018.

[16] Z. Wu and T. Alkhalifah, "A new wave equation based source location method with full-waveform inversion," in Proc. 74th Annu. Int. Conf. Exhib., EAGE, Extend Abstr., 2017, Paper Th P6 15.

[17] C. Song, Z. Wu, and T. Alkhalifah, "Passive seismic event estimation using multiscattering waveform inversion," Geophysics, vol. 84, no. 3, pp. KS59-KS69, 2019.

[18] C. Song, T. Alkhalifah, and Z. Wu, "Microseismic event estimation and velocity analysis based on a source-focusing function," Geophysics, vol. 84, no. 3, pp. KS85-KS94, 2019.

[19] C. Willacy et al., "Full-waveform event location and moment tensor inversion for induced seismicity," Geophysics, vol. 84, no. 2, pp. KS39-KS57, 2019.

[20] C. Willacy et al., "Application of full-waveform event location and moment-tensor inversion for groningen induced seismicity," Leading Edge, vol. 37, no. 2, pp. 92-99, 2018.

[21] A. Tarantola, "Inversion of seismic reflection data in the acoustic approximation," Geophysics, vol. 49, pp. 1259-1266, 1984.

[22] T. V. Leeuwen and F. Herrmann, "Mitigating local minima in fullwaveform inversion by expanding the search space," Geophys. J. Int., vol. 195, pp. 661-667, 2013.

[23] T. Alkhalifah, "Linear wavefield optimization using a modified source: Communications in Computational Physics," 2019, doi: 10.4208/cicp.OA2018-0087.

[24] T. Alkhalifah and C. Song, "An efficient wavefield inversion," in Proc. 81th EAGE Conf. Exhib., 2019.

[25] R. E. Plessix, "A review of the adjoint-state method for computing the gradient of a functional with geophysical applications," Geophysical J. Int., vol. 167, pp. 495-503, 2006.

[26] H. Wang, Q. Guo, T. Alkhalifah, and Z. Wu, "Regularized passive elastic full-waveform inversion with an unknown source," in Proc. SEG Tech. Program Expanded Abstr., 2018, pp. 2932-2936.

[27] G. Huang and W. Symes, "Matched source waveform inversion: space-time extension," in Proc. 86th Annu. Int. Meet., SEG, Expanded Abstr., 2016, pp. $1426-1431$.

[28] T. Alkhalifah and C. Song, "An efficient wavefield inversion: Utilizing a modified source function in the wave equation," Geophysics, vol. 84, no. 6, pp. 1-70, 2019.

[29] T. Alkhalifah and Z. Wu, "Multiscattering inversion for low-model wavenumbers," Geophysics, vol. 81, no. 6, pp. R417-R428, 2016. 